Trans

continentales
Transcontinentales

Sociétés, idéologies, système mondial

$1 \mid 2005$

Réforme et « Grand Moyen-Orient »

\title{
Épistémologie : les savoirs du lointain
}

Alain MAHÉ et Kmar BENDANA (dir.), Savoirs du lointain et sciences sociales, Paris, Éditions Bouchene, 2004, 272 p.

Jean-Luc Racine

\section{OpenEdition}

Journals

Édition électronique

URL : http://journals.openedition.org/transcontinentales/1402

DOI : 10.4000/transcontinentales. 1402

ISBN : 978-2-8218-1410-3

ISSN : $1775-397 X$

Éditeur

Editions de la maison des sciences de l'homme

Édition imprimée

Date de publication : 31 décembre 2005

Pagination : 141-143

ISBN : 2200-92168-3

ISSN : 1950-1684

\section{Référence électronique}

Jean-Luc Racine, «Épistémologie : les savoirs du lointain », Transcontinentales [En ligne], 1 | 2005,

document 10, mis en ligne le, consulté le 25 septembre 2020. URL : http://journals.openedition.org/ transcontinentales/1402 ; DOI : https://doi.org/10.4000/transcontinentales.1402

Ce document a été généré automatiquement le 25 septembre 2020.

Tous droits réservés 


\title{
Épistémologie : les savoirs du lointain
}

\author{
Alain MAHÉ et Kmar BENDANA (dir.), Savoirs du lointain et sciences \\ sociales, Paris, Éditions Bouchene, 2004, 272 p.
}

Jean-Luc Racine

\section{RÉFÉRENCE}

Alain MAHÉ et Kmar BENDANA (dir.), Savoirs du lointain et sciences sociales, Paris, Éditions Bouchene, 2004, 272 p.

1 Trois jours de dialogue initiés par l'Institut de recherche sur le Maghreb contemporain, basé à Tunis, et l'EHESS nous valent cet ouvrage remarquable, publié chez un éditeur discret, mais que devraient lire tous ceux qui pratiquent les « savoirs du lointain », ou qui s'y intéressent. Le titre est ambigu, bien sûr, ou historiquement situé. Sont interrogées ici les aires culturelles, ces catégories (parfois critiquées) des sciences sociales occidentales de l'ailleurs, dans un échange où prennent leur part des chercheurs tunisiens, algériens et marocains. Pour eux, le lointain des autres est leur proche, leur quotidien. Déjà se dessine une question essentielle: en s'exportant, les sciences sociales imposent-elles des références conceptuelles qui enfermeraient les praticiens locaux de la recherche dans des cadres inadéquats, ou incomplets?

2 En publiant Peaux noires, masques blancs en 1952, Franz Fanon donnait sa réponse, avant de rejoindre le Front de libération nationale algérien quatre ans plus tard. Dans son analyse du « dilemme de la construction de la sociologie au Maghreb », Lilia ben Salem montre finement comment, dans le cadre post-colonial, les choses se compliquent plus encore. Au départ, la sociologie maghrébine des années 1960 est « fortement liée aux projets de développement initiés au lendemain des indépendances ». Portée par une volonté de construction nationale, elle donne une attention prioritaire au travail de terrain, « en occultant purement et simplement les savoirs de la période coloniale ». Elle perd ainsi « l'occasion d'exercer un regard critique sur des interprétations de la société 
qui, aussi orientées qu'elles fussent, auraient pu permettre de dévoiler les continuités au-delà des ruptures, de formuler des hypothèses pour analyser le changement social, de réfuter des thèses pour en construire de nouvelles ». Vient ensuite de temps des réajustements, dans un double va-et-vient. D'une part, la volonté de réinsérer la sociologie maghrébine dans le champ scientifique international pousse parfois à manquer « de distance critique à l'égard des outils importés ». De l'autre - parfois chez les mêmes auteurs - on met l'accent sur la spécificité culturelle des sociétés étudiées, dans leur dimension arabe ou dans leur dimension musulmane, en invoquant en particulier Ibn Khaldoun et sa "science des civilisations ", 'ilm-al-'umran.

Alain Roussillon élargit le débat, en s'interrogeant pour sa part sur les modes de dépassement de l'orientalisme, dans une contribution intitulée «Les mondes d'islam : une aire culturelle virtuelle?» Il évoque l'espoir des progressistes pour qui l'orientalisme devait trouver son propre dépassement, dès lors que les "néoorientalistes " décideraient de "s'ouvrir enfin aux sciences sociales et de faire leur jonction avec la lutte des peuples pour leur émancipation ", afin de retrouver ainsi, hors de l'idéologie coloniale, "la "vérité" des sociétés orientales». Il rappelle aussi, deux ans après la publication d'Orientalism par Edward Saïd, le dossier consacré à l'islam par les Annales (XXXV, 3-4, 1980), qui pose la permanence d'un objet, l'islam dans l'espace et dans la durée, et la permanence d'un sujet, homo islamicus, pour chercher « une sorte de juste milieu entre "deux dangers symétriques : les proclamations sur la spécificité de l'islam qui le rendraient opaque aux chercheurs venant des autres cultures et inaccessible aux autres sciences sociales, comme les illusions d'une histoire universelle dans laquelle se noieraient les différences culturelles, [qui] conduisent les unes et les autres à une impasse" ». Trois protocoles sont identifiés pour aller plus loin. D'abord, clarifier les relations entre le Texte fondateur de l'islam, «l'être au monde spécifique des musulmans » qu'il inspire, et, par ailleurs, « les systèmes de solidarité et de compétition caractéristiques de toute socialité humaine ». Ensuite, faire porter l'interrogation sur le champ politique. S'il est dit que, dans le Dâr al-islam, le religieux et le politique sont inséparables, «toute science politique possible des sociétés musulmanes se voit assigner pour objet ce qui les constitue comme telles - c'est-à-dire comme adéquatement ou non adéquatement musulmanes - et qui pourrait, le cas échéant, les faire cesser de l'être: terrain et aliment des débats en cours sur les conditions de la modernisation de la société musulmanes ». La problématique de la réforme abordée dans le dossier ouvrant ce numéro de Transcontinentales se nourrit pour partie de cette question. Enfin, troisième protocole, éclairer par un travail sociologique et anthropologique "le système des écarts entre l'ordre de l'idéal et celui des rapports de force effectifs", en d'autres termes, "opérer le passage d'une représentation holiste de la société, enracinée dans le rapport au mythe fondateur de la cité de Dieu sur terre, à l'analyse segmentaire de l'organisation des systèmes d'interactions qui constituent les sociétés concrètes ». Et Roussillon de conclure à la nécessité, pour tous les spécialistes des sociétés musulmanes, de résister à la fascination du même, à la fascination de l'islam dont parlait Maxime Rodinson, pour ne pas «se laisser enfermer par le discours des acteurs et par les catégories à travers lesquelles ceux-ci se perçoivent et énoncent le sens du vivre ensemble».

4 L'un des grands mérites de cet ouvrage n'est pas seulement de reprendre, dans un dialogue trans-méditerranéen, la question de l'orientalisme. C'est aussi d'ouvrir au plus large la problématique des aires culturelles : Afrique, Inde, Océanie, mais aussi les ÉtatsUnis et leurs "études américanistes", la France des années 1930 et son «folklore 
républicain ", la Russie au tournant du vingtième siècle, le Japon en quête de soi, font l'objet de chapitres dont il faudrait également rendre compte. Manque, et c'est dommage, une étude en miroir de l'Occident, étudié (trop peu) par des chercheurs en sciences sociales venus «du lointain». Mais ce riche ouvrage soulève des questions essentielles. On y parle beaucoup de l'histoire des idées et des représentations de l'Autre, au fil des temps coloniaux et post-coloniaux. Cette histoire, c'est aussi celle du temps présent, car la recherche d'aujourd'hui n'a pas clos pour de bon la dualité entre ce que, dans sa postface, Francis Zimmerman appelle « deux types de savoirs du lointain qui sont en concurrence depuis le XIX' siècle : l'érudition (l'orientalisme) et l'ethnographie (les sciences sociales) ». Autre actualité : la critique des essentialismes, lancée voici un quart de siècle, est toujours de rigueur, car sous des formes molles ou radicales, clichés xénophobes « soft » ou fondamentalismes de toutes obédiences, l'essentialisme n'est pas mort, et irrigue toujours les débats (para)politiques et les visions de l'Autre, la mondialisation jouant en l'affaire tantôt un rôle de passeur, tantôt, à front renversé, celui de fourrier des crispations identitaires, pour de bonnes ou de mauvaises raisons.

Enfin, cet ouvrage nous rappelle que tous les « savoirs du lointain », d'une aire à l'autre, ne sont pas similaires, selon la place qu'y tiennent les sciences sociales, selon le rôle qu'y jouent les chercheurs autochtones. Il nous montre aussi, avec Alain Mahé, que le projet orientaliste était au départ un projet universaliste, se donnant pour but "d'élargir les références de l'humanisme européen en promouvant d'autres cultures classiques ", ce qui n'empêcha pas "le savoir des lointains " d'opposer rapidement l'«humanité sauvage» aux "humanités classiques», et de souligner le poids des spécificités. Dans ce contexte, le balancement entre universalisme et particularisme est fort différent de la dialectique du global et du local, aujourd'hui de rigueur. Mais il serait éclairant de retrouver ce qui, du premier diptyque, a percolé dans le second. Ce n'est pas l'un des moindres mérites de cet ouvrage érudit que d'ouvrir ainsi des pistes pour mieux comprendre le présent, ou du moins, pour mieux l'interroger. Kmar Bendana et Jacques Revel, en donnant au terme de "bricolage" une connotation créative, nous le disent à leur façon: l'interrogation permanente vaut mieux que les fausses certitudes. Les crises les plus violentes de l'histoire immédiate ne nous le rappellent que trop.

\section{AUTEURS}

\section{JEAN-LUC RACINE}

Centre d'études de l'Inde et de l'Asie du Sud 